\title{
Comparison of videolaryngoscopy and direct laryngoscopy by German paramedics during out-of- hospital cardiopulmonary resuscitation. An observational prospective study.
}

Joachim Risse ( $\nabla$ joachim.risse@uk-essen.de)

Universitatsklinikum Essen https://orcid.org/0000-0002-1671-8506

Christian Volberg

Philipps-Universitat Marburg

Thomas Kratz

Philipps-Universitat Marburg

Birgit Plöger

Philipps-Universitat Marburg

Andreas Jerrentrup

Philipps-Universitat Marburg

Dirk Pabst

Uniklinik Essen

Clemens Kill

Uniklinik Essen

Research article

Keywords: Cardiopulmonary resuscitation, Endotracheal intubation, Paramedics, Out-of-hospital cardiac arrest, Videolaryngoscopy

Posted Date: February 10th, 2020

DOI: https://doi.org/10.21203/rs.2.16606/v3

License: (c) (i) This work is licensed under a Creative Commons Attribution 4.0 International License.

Read Full License

Version of Record: A version of this preprint was published at BMC Emergency Medicine on March 23rd, 2020. See the published version at https://doi.org/10.1186/s12873-020-00316-z. 


\section{Abstract}

Introduction: Videolaryngoscopy (VL) has become a popular method of intubation (ETI). Although VL may facilitate ETI in less-experienced rescuers there are limited data available concerning ETI performed by paramedics during CPR. The goal was to evaluate the impact VL compared with $D L$ on intubation success and glottic view during CPR performed by German paramedics. We investigated in an observational prospective study the superiority of VL by paramedics during CPR compared with direct laryngoscopy (DL).

Methods: In a single Emergency Medical Service (EMS) in Germany with in total 32 ambulances paramedics underwent an initial instruction from in endotracheal intubation (ETI) with GlideScope ${ }^{\circledR}(\mathrm{GVL})$ during resuscitation. The primary endpoint was good visibility of the glottis (Cormack-Lehane grading 1/2), and the secondary endpoint was successful intubation comparing GVL and DL.

Results: In total $n=97$ patients were included, $n=69$ with $D L(n=85$ intubation attempts) and $n=28 \mathrm{VL}$ ( $n=37$ intubation attempts). Videolaryngoscopy resulted in a significantly improved visualization of the larynx compared with $\mathrm{DL}$. In the group using GVL, $82 \%$ rated visualization of the glottis as CL $1 \& 2$ versus $55 \%$ in the DL group ( $p=0.02$ ). Despite better visualization of the larynx, there was no statistically significant difference in successful ETI between GVL and DL (GVL 75\% vs. DL 68.1\%, p = 0.63).

Conclusions: We found no difference in Overall and First Pass Success (FPS) between GVL and DL during CPR by German paramedics despite better glottic visualization with GVL. Therefore, we conclude that education in VL should also focus on insertion of the endotracheal tube, considering the different procedures of GVL.

\section{Introduction}

Maintaining an open airway is one of the most important procedures in emergency care during advanced life support (ALS) resuscitation and is essential for adequate ventilation of the patient. Emergency medical services (EMS) in Germany is designed as a two-tiered system including a physician-staffed EMS unit in all life-threatening cases and an ALS-Ambulance with paramedics. As soon as the emergency physician is on scene, procedures such as endotracheal Intubation (ETI) are performed by the physician. Due to the higher availability of paramedic-staffed ambulances, in many cases the paramedics are on scene before the arrival of the EMS physician. Although paramedics are trained in ALS including direct laryngoscopy and endotracheal intubation, the rate of performing endotracheal intubation by paramedics before the arrival of the emergency physician unit is low. Therefore we investigated the effect of $\mathrm{VL}$ compared to DL by paramedics during CPR before arrival of the emergency physician on both visibility of the glottis and intubation success rate.

Adequate ventilation, improved oxygenation, and avoidance of aspiration are important factors concerning the rate of ROSC as well as the neurological outcome of a patient undergoing $\operatorname{CPR}(1,2)$. 
Current updated international recommendations for advanced airway management from the International Liaison Committee on Resuscitation (ILCOR) suggest supraglottic devices for adults with Out-of-hospital cardiac arrest (OHCA) in settings with a low intubation success rate (3). In case of less experienced providers they recommended mask ventilation or supraglottic devices in order to not interrupt chest compressions.In the Anglo-American paramedic system, the success rates for prehospital ETI using DL are between $71 \%$ and $75 \%(4,5)$. In contrast to Anglo-American paramedic system to For German paramedics, ETI during CPR is generally a rare event as the attending physician usually carries out the procedure. Some investigators have shown that untrained users have a $51 \%$ rate of successful intubation with DL (Maclntosh) (6). In contrast, with VL inexperienced users have an especially steep learning curve and a significantly higher success rate $(6,7)$. Videolaryngoscopy is superior to DL when the first attempt at intubation has failed and is associated with a reduction in esophageal intubations $(8,9)$.

Current studies could demonstrate that VL improves glottic opening but do not improve First Pass Success (FPS) (10-12). On the other Hand there is valid data that VL improves FPS (13-15). Moreover, data exist showing that the overall success rate of ETI by inexperienced physicians during CPR is significantly higher with $\mathrm{VL}$ than with $\mathrm{DL}(4,9,16)$. During CPR with ongoing chest compression using $\mathrm{VL}$ for ETI might result in reduced interruption of chest compressions (17-19). Several studies have shown that in pre-hospital settings there is an alarmingly high rate of failed ETI, especially when performed by non-physicians (20-22).

We investigated the impact of using videolaryngoscopy $(\mathrm{VL})$ instead of direct laryngoscopy (DL) by paramedics in out-of-hospital cardiac arrest before arrival of the emergency physician on scene in a semirural county in Germany. We focused our investigation on glottic opening and overall und First Pass success (FPS).

\section{Methods}

\section{Study Design and Time period}

With approval by the institutional ethics committee (EudraCT number 2015-000919-41, retrospectively registered), we designed a prospective observational study comparing DL and GVL by paramedics in outof-hospital cardiac arrest (OHCA) without an emergency physician on scene. We performed our investigation under actual field conditions over a period of 4 years to include a sufficient number of cases. ETI should be performed either with VL (GlideScope® Ranger, GVL) or the standard DL (Maclntosh) depending on the availability of VL at time of CPR. Therefore four GVL devices were allocated on four of the 32 ambulances for six-month to assure experience and rotated after six month to the next four ambulances of the EMS agency. The rotation of the $4 \mathrm{GVL}$ continued until the end of the investigation period of 4 years.

Study Setting and Population 
The inclusion criteria were patients in non-traumatic cardiac arrest, ongoing basic life support (BLS) with chest compressions and bag-mask-ventilation, and absence of an emergency physician on scene.The exclusion criteria were patients aged less than 12 years, the presence of an emergency physician on scene, and the primary use of a supraglottic airway device by the paramedics.

All paramedics of EMS received a training course explaining handling of GVL before starting the study. For this purpose, a manikin exercise phantom head was used for ETI training, and the correct and different handling of the GVL was practiced under medical supervision, ca. 20 intubations (JR, TK, CK). A new standard operating procedure (SOP) "Airway management with videolaryngoscopy (GVL)" was developed before commencing the study and was implemented in the annual paramedic training. The content of the SOP was: If the ambulance was equipped with GVL it was mandatory using first line GVL for securing the airway during ALS procedure without EMS physician on scene. The new SOP and an instruction manual, with instructions for the different technique and preparation of the tube with a rigid stylet for hyper angulated blades, were made available to all paramedics via the company's intranet. After Training period paramedics had the opportunity to practice on a manikin with medical supervision during the six month of availability of the GVL device at their EMS base. There was no additional training in patients as in the operation theatre.

Our primary endpoint was the visibility of the glottis with a Cormack-Lehane (CL) score of 1 or 2, and the secondary endpoint was the overall ETI success and the First Pass Success FPS rate during out-ofhospital CPR.

An ETI attempt was defined whenever a VL or DL blade passed the teeth to intubate and secure the airway during advanced cardiac life support (ACLS). The number of required attempts was recorded. Successful ETI was defined as the successful placement of an endotracheal tube and correct pulmonary ventilation with a positive capnography. The Success with a positive EtCO2 waveform was confirmed by the EMS physician arriving on scene. A maximum of two attempts were allowed per the internal ALS protocol of the EMS. If ETI failed, paramedics were recommended to use a laryngeal mask to secure the airway. After every ETI attempt during CPR by paramedics, the research team sent a self-report questionnaire to the paramedic team to acquire data.

\section{Outcome Measures and Data Analysis}

Non-normally distributed variables were expressed as the median and upper and lower quartiles (Q.25 and Q.75). These data were analyzed by using the Mann-Whitney $\mathrm{U}$ test. Data were also presented as percentages with confidence intervals $(\mathrm{Cl})$. Differences in frequency were tested for significance using the chi-square test and for small cell occupations using Fisher's exact test. To test for correlations, the point biserial correlation coefficient (r.pb) was used. The significance level was set to alpha $=0.05$. Data are presented as histograms and box-and-whisker diagrams. All statistical calculations were performed using the statistics packages SPSS (version 22) and BiAS for Windows (version 11.03, Epsilon Verlag, 2016). 


\section{Results}

In total 134 case report forms (CRFs) from patients after CPR were collected. For various reasons, $37 \mathrm{CRF}$ were excluded from further evaluation (see Figure 1). Finally, the CRF`s of $n=97$ patients were included, $n$ $=69$ with use of $\mathrm{DL}$ (with $\mathrm{n}=85$ intubation attempts) and $\mathrm{n}=28$ with use of GVL (with $\mathrm{n}=37$ intubation attempts). Paramedics' professional experience in years (7yrs in $\mathrm{DL}$ vs 6.5yrs in $\mathrm{GVL}, \mathrm{p}=0.48$ ) and the estimated number of conventional (DL) intubations ( $n=30$ DL vs $n=22.5 \mathrm{GVL}, p=0.14$ ) performed previously by the paramedics were similar in both groups. For the $\mathrm{CL}$ grade $1-4$, a statistically significant difference in our data could be shown between the two groups $(p=0.002)$ (see Figure 2$)$. With GVL a CL grade of 1 was significantly more frequent, with a difference of $27.7 \%$ compared with $D L(p=0.004)$ (see Table 1). The proportion of dichotomized CL grades $1 \& 2$ vs $3 \& 4$ were statistically significantly different in the GVL group compared with the DL group. CL grades $1 \& 2$ represent an easier ETI in contrast to Grades 3\&4, which represent a more difficult ETI. CL grade $1 \& 2$ was statistically significantly more frequent in the GVL group than in the DL group $(p=0,02)$ (see Table 1). Regardless of the method used for an ETI by paramedics, our data showed that the number of unsuccessful ETIs increased with a higher CL grade $($ r.pb $=0.614, p<0.0001)($ see Table 2).

The other focus of the investigation (overall ETI success and First Pass success FPS) did not differ significantly between the GVL group and the DL group $(p=0,63)$ (see Table 3 ). There was no statistically significant difference between the two groups in the number of intubation attempts (1 or 2), $(p=0.4)$ (see Table 4). Also, the success rate on the first and second attempt was similar in both groups. The difference found was not significant $(p=0.2)$ (see Table 5).

\section{CL-Grade GVL DL p-value}

\begin{tabular}{llll}
\hline I & $11(39,29 \%)$ & $8(11,59 \%)$ & 0,004 \\
II & $12(42,86 \%)$ & $30(43,48 \%)$ & 1 \\
III & $3(10,71 \%)$ & $20(28,99 \%)$ & 0,07 \\
IV & $2(7,14 \%)$ & $11(15,94 \%)$ & 0,34
\end{tabular}




\section{Dichotomized CL-Grade GVL DL p-value \\ $\begin{array}{llll}\text { I+II (easier ETI) } & 23(82,15 \%) & 38(55,07 \%) & 0,02\end{array}$ \\ III+IV (difficult ETI) $\quad 5(17,85 \%) \quad 31(44,93 \%) \quad \mathbf{0 , 0 2}$}

Table 1. Comparison View of the larynx by Cormack-Lehane classification system videolaryngoscopy (GVL) vs. direct laryngoscopy (DL) 
CL-Grade

Total no. ETI successful

Total no. ETI unsuccessful
I

$17(89,5 \%)$

$2(10,5 \%)$
II

37

$(88,1 \%)$
III

12

IV

2

point biserial correlation coefficient r.pb $=0,614, \mathrm{p}<0,0001$

Table 2. Comparison Total endotracheal intubation (ETI) success depending on the Cormack-Lehane classification system

Table 3. Comparison of Overall Successful Endotracheal Intubation (ETI)

ETI successful ETI unsuccessful

\begin{tabular}{c|cc|c}
\hline GVL & $21(75 \%)$ & $7(25 \%)$ & 28 \\
\multirow{2}{*}{ DL } & $47(68.1 \%)$ & $22(31.9 \%)$ & 69 \\
\cline { 2 - 4 } & 68 & 29 & 97 \\
\hline & \multicolumn{2}{|c|}{ Fisher's exact test two-tailed $P$-value is 0.626777 } \\
\hline
\end{tabular}

Table 4. Comparison of the Number of Endotracheal Intubation (ETI) Attempts

\begin{tabular}{|c|c|c|c|}
\hline & One ETI attempt & Two ETI attem & \\
\hline GVL & $19(67.9 \%)$ & $9(32.1 \%)$ & 28 \\
\hline \multirow[t]{2}{*}{$\mathrm{DL}$} & $53(76.8 \%)$ & $16(23.2 \%)$ & 69 \\
\hline & 72 & 25 & 97 \\
\hline & \multicolumn{3}{|c|}{ Fisher's exact test two-tailed $P$-value is 0.4434} \\
\hline
\end{tabular}

Table 5. Comparison of the Success Rate on the 1st and 2nd Attempt 


\begin{tabular}{c|cc|c}
\hline \multirow{2}{*}{} & First attempt ETI success & Second attempt ETI success \\
\hline GVL & $15(71.4 \%)$ & $6(28.6 \%)$ & 21 \\
DL & $40(85.1 \%)$ & $7(14.9 \%)$ & 47 \\
\cline { 2 - 4 } & 55 & 13 & 68 \\
\hline
\end{tabular}

Fisher's exact test two-tailed $P$-value is 0.199879

\section{Discussion}

Paramedics with limited experience in both $D L$ and videolaryngoscopic ETI might have improved success rates using GVL as a first-line device in emergency airway management with CPR. Our results from this out-of-hospital observational study demonstrate improved visualization of the larynx with GVL. Despite better visualization of the larynx with GVL, the first pass success FPS and the overall success rates for ETI were not improved compared with DL during CPR when performed by German paramedics. This may be due to less experience in handling a videolaryngoscope and infrequent opportunities for German paramedics to perform ETI in general.

Previous investigations showed a significantly higher intubation success rate by inexperienced users during CPR with GVL than with DL $(16,19)$. Lee et al. investigated tracheal intubation during in-hospital cardiopulmonary resuscitation (16). These results from clinical research cannot simply be transferred to the out of hospital setting. However we were not able to show an increased success rate for ETI when performed by German paramedics who are less experienced in the procedure.

Endotracheal intubation during resuscitation is frequently associated with a difficult airway and shows FPS with VL, depending on the study, between $73 \%$ and $94 \%$, even for experienced physicians $(16,17)$. Most of the previous studies observing GVL during CPR investigated experienced physicians or were just simulation studies with mannequins $(17,23-25)$. The differences to our results might be based on user experience (physicians, non-physicians) with the procedure. We suspect a broad range of user experiences across individuals and studies.

Ducharme et al. saw similar relevant results in their investigation of American paramedics over a period of 34 months. The group showed that VL had similar FPS rates and even better laryngoscopic visualization compared with DL. They used the King Vision® ${ }^{\circledR}$ videolaryngoscope, whereas our investigation used the GlideScope ${ }^{\circledR}$ Ranger (26). In addition, our study results showed a trend towards a higher rate of successful ETI on the second attempt with GVL. This might be based on an immediate learning process from the first attempt to the second attempt with VL. A minimal optimization during the 
second attempt (blood and secretion suction, cleaning the lens, view of the monitor) might be enough in such a situation to successfully intubate with GVL. Nouruzi-Sedeh et al. showed a success rate of more than $90 \%$ on the first attempt in their investigation with personnel untrained in intubation using GVL. In the second attempt, all subjects were successfully intubated with the GlideScope technique (6). In this context, due to the small number of cases, we could only see a statistically insignificant trend in our data towards a higher rate of successful ETI on the second attempt with GVL during CPR.

During out-of-hospital CPR there are multiple external influences and stressors on the paramedic team, for example, the unfamiliar environment, lighting etc. Russo et al. postulate that videolaryngoscopes are helpful for emergency intubations, but sufficient experience in dealing with the devices is essential. They also showed the limitations of videolaryngoscopes, e.g. blood, vomit or secretions in front of the lens, as well as bright light producing glare on the screen (27). These stressors might also be responsible for the poor performance observed with both devices.

\section{Limitations}

The first limitation of our study is related to its design. We performed a preliminary observation trial with paramedics from single EMS area. For that reason, our study sample was small and unbalanced. For paramedics in Germany, ETI is a rare event, and we performed our investigation under actual field conditions over a period of 4 years to include a sufficient number of cases. In most cases of pre-hospital emergency medicine in Germany, an emergency physician performs intubation. To obtain a larger case number in an adequate investigation time period, several different EMS should be included in further investigations. All paramedics were instructed to report during the investigation period. There was no cross-checking how many patients underwent ETI by paramedics without a corresponding CRF returned. In addition, there is a possibility of reporting bias. Despite anonymization of the questionnaires and optional participation for the paramedics in this investigation, positive results and positive occurrences might be reported more often than negative ones. The instruction for the paramedics in using GVL instead of DL was only a manikin training without additional training in patients in elective surgery, e.g. All paramedics underwent training in DL in their professional education much more intensively than training of GVL for this study, so there might be a bias in favour of $D L$ as a limitation of this study.

A further limitation is due to the different levels of training with $D L$ and GVL of the individual paramedics in the single investigated EMS. Based on the variability of individual intubation experience among paramedics in this single EMS, the results cannot be transferred to another EMS. Furthermore, our study was conducted over 4 years and there has possibly been an increase in SGA use by paramedics, because more recent studies indicated that SGAs could be equivalent or better than ETI $(28,29)$. This might have also an effect on the small number of cases in the whole investigation period.

We used the GlideScope ${ }^{\circledR}$ Ranger videolaryngoscope in our investigation, while the group of Ducharme et al. for example used the King Vision ${ }^{\circledR}$ videolaryngoscope (26). The two studies obtained similar results; however, there are currently many different videolaryngoscopes with varying designs and quality 
available on the market. For these reasons, our study results should not be generalized, and further investigation is needed.

\section{Conclusion}

We found no difference in Overall and First Pass Success FPS between GVL and DL during CPR by German paramedics despite better glottic visualization with GVL.

Therefore, we conclude that more training in VL for German paramedics is needed and education in VL should focus on insertion of the endotracheal tube, considering the different procedures of GVL.

\section{List Of Abbreviations}

ACLS - Advanced cardiac life support

ALS - Advanced life support

CL grading - Cormack-Lehane grading

CPR - Cardiopulmonary resuscitation

CRF - Case report form

CK - Clemens Kill

EMS - Emergency medical services

ETI - Endotracheal intubation

DL - Direct laryngoscopy

FPS -First Pass Success

GVL - GlideScope ${ }^{\circledR}$ videolaryngoscopy

ILCOR - International Liaison Committee on Resuscitation

JR - Joachim Risse

OHCA - Out-of-hospital Cardiac Arrest

ROSC - Return of spontaneous circulation

SOP - Standard operating procedure

TK - Thomas Kratz 
VL - Videolaryngoscopy

\section{Declarations}

\section{Ethics approval and consent to participate}

Ethics approval was granted by the Ethics Committee of Philipps-University Marburg, Baldingerstrasse/Postfach 2360, 35032 Marburg, Germany Az.: 184/10, 10.11.2010.

Consent to participate was not required. This investigation in the context of resuscitation requires no written consent of the resuscitated patients in the context of quality assurance. The "Ordinance on Quality Assurance in Rescue Services" of 27 February 2003 (GVBI. I p. 105), issued based on section 26 (1), (3) and (4) and section 27 (1) of the Hessian Rescue Service Act 1998 of 24 November 1998 (GVBI. I p. 499) explicitly commits the institutions responsible for emergency care within the rescue service to anonymous data collection and evaluation in the context of quality assurance. The inclusion of clinical follow-up data in conjunction with Section 12 (2) no. 8 of the Hessian Hospital Act 2002 of 6 November 2002 (GVBI. I p. 662) is also finally regulated. With reference to this legal basis, the Ethics in Medicine Commission of the Philipps-University of Marburg agreed in 2010 to the investigation.

\section{Consent for publication}

Not applicable

\section{Availability of data and material}

The data that support the findings of this study are available from the corresponding author. The datasets used and analyzed during the current study are available from the corresponding author on reasonable request.

\section{Competing interests}

The authors declare that they have no competing interests.

\section{Funding}

No funding

\section{Authors' contributions}

$\mathrm{JR}$ and $\mathrm{CV}$ analyzed and interpreted the patient data. JR and CK were the major contributors in writing the manuscript. CV, TK, BP, AJ, DP were involved in data acquisition and data analysis, read and approved the final manuscript.

Acknowledgments 
Comesta (Consulting Methods \& Statistics), Dipl.-Psych. Thomas Ploch for his statistical support.

The authors report no conflicts of interest

\section{References}

1. Spindelboeck W, Schindler O, Moser A, Hausler F, Wallner S, Strasser C, et al. Increasing arterial oxygen partial pressure during cardiopulmonary resuscitation is associated with improved rates of hospital admission. Resuscitation. 2013;84(6):770-5.

2. Yeh ST, Cawley RJ, Aune SE, Angelos MG. Oxygen requirement during cardiopulmonary resuscitation (CPR) to effect return of spontaneous circulation. Resuscitation. 2009;80(8):951-5.

3. Soar J, Maconochie I, Wyckoff MH, Olasveengen TM, Singletary EM, Greif R, et al. 2019 International Consensus on Cardiopulmonary Resuscitation and Emergency Cardiovascular Care Science With Treatment Recommendations: Summary From the Basic Life Support; Advanced Life Support; Pediatric Life Support; Neonatal Life Support; Education, Implementation, and Teams; and First Aid Task Forces. Circulation. 2019;140(24):e826-e80.

4. Myers LA, Gallet CG, Kolb LJ, Lohse CM, Russi CS. Determinants of Success and Failure in Prehospital Endotracheal Intubation. West J Emerg Med. 2016;17(5):640-7.

5. Voss S, Rhys M, Coates D, Greenwood R, Nolan JP, Thomas M, et al. How do paramedics manage the airway during out of hospital cardiac arrest? Resuscitation. 2014;85(12):1662-6.

6. Nouruzi-Sedeh P, Schumann M, Groeben H. Laryngoscopy via Macintosh blade versus GlideScope: success rate and time for endotracheal intubation in untrained medical personnel. Anesthesiology. 2009;110(1):32-7.

7. Sakles JC, Mosier J, Patanwala AE, Dicken J. Learning curves for direct laryngoscopy and GlideScope $(R)$ video laryngoscopy in an emergency medicine residency. West J Emerg Med. 2014;15(7):930-7.

8. Sakles JC, Javedani PP, Chase E, Garst-Orozco J, Guillen-Rodriguez JM, Stolz U. The Use of a Video Laryngoscope by Emergency Medicine Residents Is Associated With a Reduction in Esophageal Intubations in the Emergency Department. Academic Emergency Medicine. 2015;22(6):700-7.

9. Sakles JC, Mosier JM, Patanwala AE, Dicken JM, Kalin L, Javedani PP. The C-MAC® Video Laryngoscope Is Superior to the Direct Laryngoscope for the Rescue of Failed First-Attempt Intubations in the Emergency Department. The Journal of Emergency Medicine. 2015;48(3):280-6.

10. Griesdale DE, Liu D, McKinney J, Choi PT. Glidescope(R) video-laryngoscopy versus direct laryngoscopy for endotracheal intubation: a systematic review and meta-analysis. Can J Anaesth. 2012;59(1):41-52.

11. Guyette FX, Farrell K, Carlson JN, Callaway CW, Phrampus P. Comparison of video laryngoscopy and direct laryngoscopy in a critical care transport service. Prehosp Emerg Care. 2013;17(2):149-54.

12. Yun BJ, Brown CA, 3rd, Grazioso CJ, Pozner CN, Raja AS. Comparison of video, optical, and direct laryngoscopy by experienced tactical paramedics. Prehosp Emerg Care. 2014;18(3):442-5. 
13. Cinar O, Cevik E, Yildirim AO, Yasar M, Kilic E, Comert B. Comparison of GlideScope video laryngoscope and intubating laryngeal mask airway with direct laryngoscopy for endotracheal intubation. Eur J Emerg Med. 2011;18(2):117-20.

14. Sakles JC, Patanwala AE, Mosier JM, Dicken JM. Comparison of video laryngoscopy to direct laryngoscopy for intubation of patients with difficult airway characteristics in the emergency department. Intern Emerg Med. 2014;9(1):93-8.

15. Su YC, Chen CC, Lee YK, Lee JY, Lin KJ. Comparison of video laryngoscopes with direct laryngoscopy for tracheal intubation: a meta-analysis of randomised trials. Eur J Anaesthesiol. 2011;28(11):78895.

16. Lee DH, Han M, An JY, Jung JY, Koh Y, Lim C-M, et al. Video laryngoscopy versus direct laryngoscopy for tracheal intubation during in-hospital cardiopulmonary resuscitation. Resuscitation. 2015;89:1959.

17. Kim JW, Park SO, Lee KR, Hong DY, Baek KJ, Lee YH, et al. Video laryngoscopy vs. direct laryngoscopy: Which should be chosen for endotracheal intubation during cardiopulmonary resuscitation? A prospective randomized controlled study of experienced intubators. Resuscitation. 2016;105:196-202.

18. Park SO, Baek KJ, Hong DY, Kim SC, Lee KR. Feasibility of the video-laryngoscope (GlideScopeß) for endotracheal intubation during uninterrupted chest compressions in actual advanced life support: $A$ clinical observational study in an urban emergency department. Resuscitation. 2013;84(9):1233-7.

19. Park SO, Kim JW, Na JH, Lee KH, Lee KR, Hong DY, et al. Video laryngoscopy improves the firstattempt success in endotracheal intubation during cardiopulmonary resuscitation among novice physicians. Resuscitation. 2015;89:188-94.

20. Cobas MA, De la Pena MA, Manning R, Candiotti K, Varon AJ. Prehospital intubations and mortality: a level 1 trauma center perspective. Anesth Analg. 2009;109(2):489-93.

21. Timmermann A, Russo SG, Eich C, Roessler M, Braun U, Rosenblatt WH, et al. The out-of-hospital esophageal and endobronchial intubations performed by emergency physicians. Anesth Analg. 2007;104(3):619-23.

22. Ufberg JW, Bushra JS, Karras DJ, Satz WA, Kueppers F. Aspiration of gastric contents: association with prehospital intubation. The American Journal of Emergency Medicine. 2005;23(3):379-82.

23. Piegeler T, Roessler B, Goliasch G, Fischer H, Schlaepfer M, Lang S, et al. Evaluation of six different airway devices regarding regurgitation and pulmonary aspiration during cardio-pulmonary resuscitation (CPR) - A human cadaver pilot study. Resuscitation. 2016;102:70-4.

24. Hossfeld B, Frey K, Doerges V, Lampl L, Helm M. Improvement in glottic visualisation by using the CMAC PM video laryngoscope as a first-line device for out-of-hospital emergency tracheal intubation: An observational study. Eur J Anaesthesiol. 2015;32(6):425-31.

25. Shin DH, Choi PC, Han SK. Tracheal intubation during chest compressions using Pentax-AWS®, GlideScope ${ }^{\circledR}$, and Macintosh laryngoscope: a randomized crossover trial using a mannequin. Canadian Journal of Anesthesia/Journal canadien d'anesthésie. 2011;58(8):733-9. 
26. Ducharme S, Kramer B, Gelbart D, Colleran C, Risavi B, Carlson JN. A pilot, prospective, randomized trial of video versus direct laryngoscopy for paramedic endotracheal intubation. Resuscitation. 2017;114:121-6.

27. Russo SG, Nickel EA, Leissner KB, Schwerdtfeger K, Bauer M, Roessler MS. Use of the GlideScope®Ranger for pre-hospital intubations by anaesthesia trained emergency physicians - an observational study. BMC Emergency Medicine. 2016;16(1):8.

28. Benger JR, Kirby K, Black S, Brett SJ, Clout M, Lazaroo MJ, et al. Effect of a Strategy of a Supraglottic Airway Device vs Tracheal Intubation During Out-of-Hospital Cardiac Arrest on Functional Outcome: The AIRWAYS-2 Randomized Clinical Trial. Jama. 2018;320(8):779-91.

29. Wang HE, Schmicker RH, Daya MR, Stephens SW, Idris AH, Carlson JN, et al. Effect of a Strategy of Initial Laryngeal Tube Insertion vs Endotracheal Intubation on 72-Hour Survival in Adults With Out-ofHospital Cardiac Arrest: A Randomized Clinical Trial. Jama. 2018;320(8):769-78.

\section{Figures}

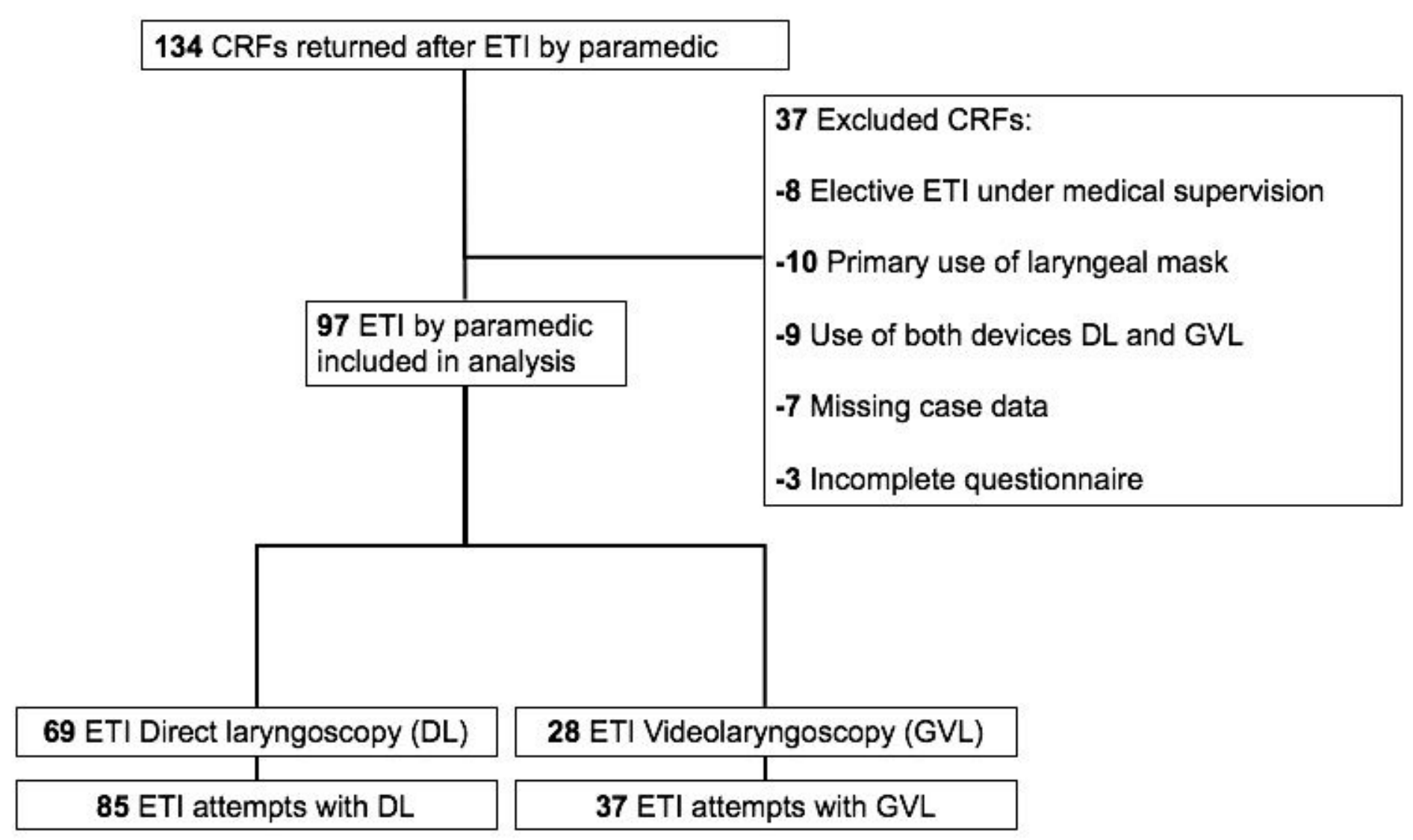

Figure 1

Diagram data evaluation 

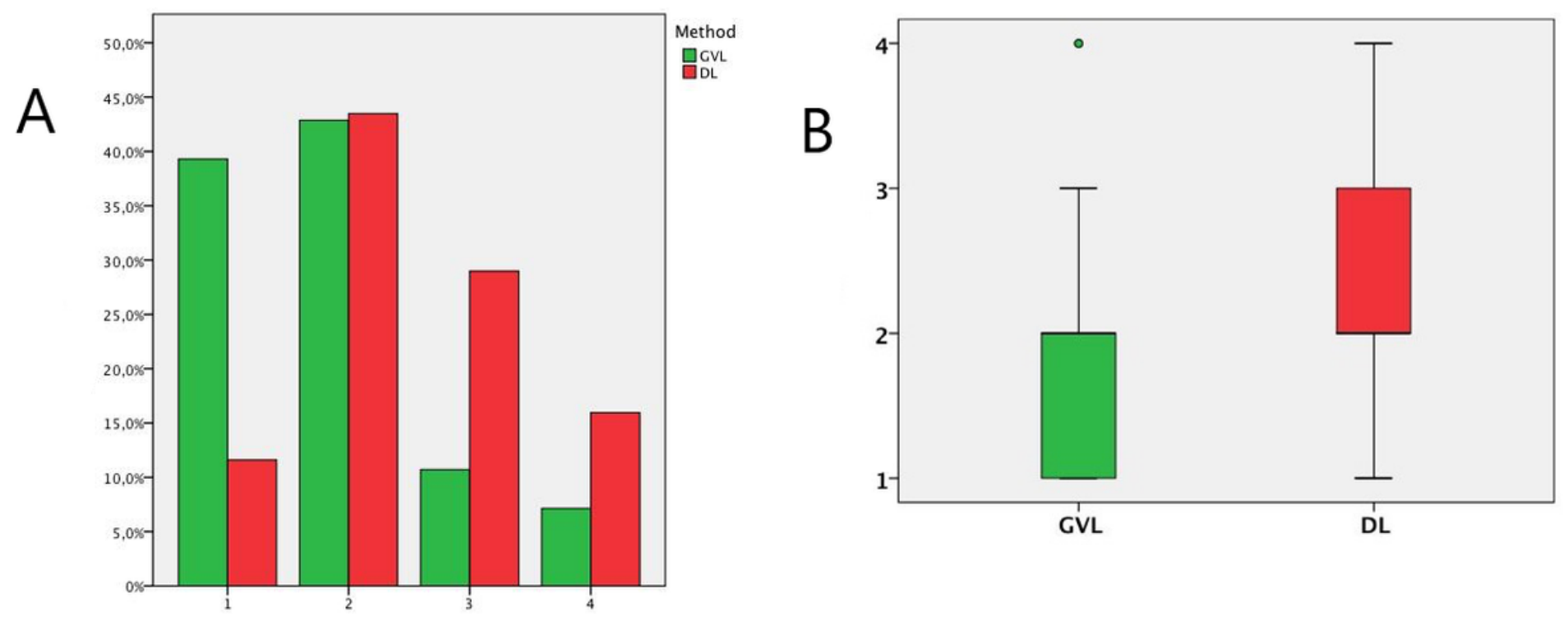

Figure 2

Frequency distribution of the Cormack and Lehane grading (CL) between videolaryngoscopy (GVL) and direct laryngoscopy (DL) Legend: 1. bar graph x-axis CL Grade, y-axis number in percent, method GVL (green) and DL (red) 2. boxplot x-axis method GVL (green) and DL (red), y-axis CL Grade 1-4

\section{Supplementary Files}

This is a list of supplementary files associated with this preprint. Click to download.

- Table15.docx 